\title{
NOTA SUI LIMITI FISIOLOGICI DELLE IMMERSIONI PROFONDE IN APNEA
}

\author{
Nota del s.c. GUIDO FERRETTI (*)
}

(Adunanza del 23 gennaio 2020)

SuNTO. - In questa nota si tratta dei limiti delle immersioni profonde in apnea nell'uomo. Dopo un breve excursus storico e una presentazione dell'evoluzione dei record di profondità, vengono presentate e discusse le teorie classiche dei limiti delle immersioni, e precisamente quella del rapporto tra capacità polmonare totale e volume residuo e quella del blood shift, che comporta aumento del volume ematico centrale Viene poi introdotta la visione attuale, basata sui principi dell'energetica dell'esercizio muscolare, che ha ribaltato la visione tradizionale, spostando la discussione su di un altro piano. Conseguenza diretta di questa nuova teoria è l'importanza di avere ampli volumi polmonari all'inizio dell'immersione, al fine di incrementare le scorte di ossigeno dell'organismo. Inoltre, si discute del ruolo del metabolismo anaerobico lattacido come possibile meccanismo di preservazione dell'ossigeno, e quindi di prolungamento delle immersioni in apnea.

$* * *$

ABSTRACT. - This article discusses the limits of deep breath-hold diving in humans. After a short historical introduction and a discussion of the evolution of depth records, the classical theories of breath-hold diving limits are presented and discussed, namely that of the ratio between total lung capacity and residual volume and that of blood shift, implying an increase in central blood volume. Then the current vision is introduced, based on the principles of the energetics of muscular exercise. The new vision has turned the classical vision upside down, moving the discussion to a different level. A direct consequence of the new theory is the importance of having large lung volumes at the start of a dive, in order to increase body oxygen stores. I finally discuss the role of anaerobic lactic metabolism as a possible mechanism of oxygen preservation, thus prolonging breath-hold duration.

(") Dipartimento di Medicina Molecolare e Traslazionale, Università di Brescia, Italy. E-mail: guido.ferretti@unibs.it 


\section{CENNI STORICI}

La pratica delle immersioni in apnea è antica quanto la civiltà umana. La prima traccia scritta di immersioni in apnea rifulge nella letteratura mitologica. Risaliamo a più di 4000 anni fa, all'epopea di Gilgamesh, che Guido Ceronetti fece pubblicare presso Adelphi in versione italiana (per chi fosse interessato, la versione integrale delle 12 tavolette cuneiformi, con traduzione verso per verso e analisi grammaticale, è scaricabile liberamente al sito https://mediterraneoantico.it/ wp-content/uploads/2018/11/Lepopea-di-Gilgamesh.pdf). In essa si narra, tra l'altro, che Gilgamesh, governatore di Uruk, sommo eroe del mito sumero, per salvare l'amico Enkidu da morte certa, parte alla ricerca della pianta che dà l'immortalità. Il saggio Utnapishtim gli rivela infine che può trovarla in una grotta sottomarina. Gilgamesh s'immerge nel lago indicatogli, la trova, la coglie, ma se la fa strappar via da un serpente sulla strada del ritorno.

Nella vita reale, la pratica si diffuse in molte terre rivierasche e insulari, allo scopo di pescare spugne, perle, frutti di mari, pesci. In molte società marine, essa era riservata alle donne. Essa si diffuse in particolare nella Ionia (Dodecanneso in particolare), in Persia e in India, in Corea e in Giappone, e nella Polinesia. Con lo sviluppo delle attività militari e la nascita delle navi da guerra, gli eserciti si dotarono di incursori sottomarini in apnea. Ne fecero uso, ad esempio, Nicia nell'assedio di Siracusa (415 a.C.) e il re fenicio di Tiro Azemilco durante l'assedio di Alessandro il Macedone (332 a.C.). Celebri divennero gli urinatores, gli incursori subacquei di Roma imperiale, un vero corpo specializzato professionale, impiegati per la prima volta da Cesare contro Pompeo nelle guerre civili, ma resi corporazione permanente dall'imperatore Claudio, che affidò loro in tempo di pace tutta la manutenzione del nuovo porto di Roma, che egli fece costruire a partire dal 42 d.C.).

In anni più recenti, in Occidente, si usarono apneisti per il recupero di tesori sommersi. Fu in questo genere di attività che i primi strumenti finalizzati ad ampliare la durata delle immersioni vennero costruiti. Il più curioso è la cosiddetta campana a immersione, che Hans Albrecht von Treileben (1625-1690) costruì nel 1664 per recuperare i cannoni e il materiale di bordo della nave da guerra Vasa, inopinatamente affondata nella rada di Stoccolma al momento del varo. Questo dispositivo (Fig. 1) consentiva di disporre per ogni immersione di una quantità d'aria ben superiore alla capacità polmonare totale dei 
sommozzatori. Una descrizione dettagliata del dispositivo e della vicenda è reperibile in [1]

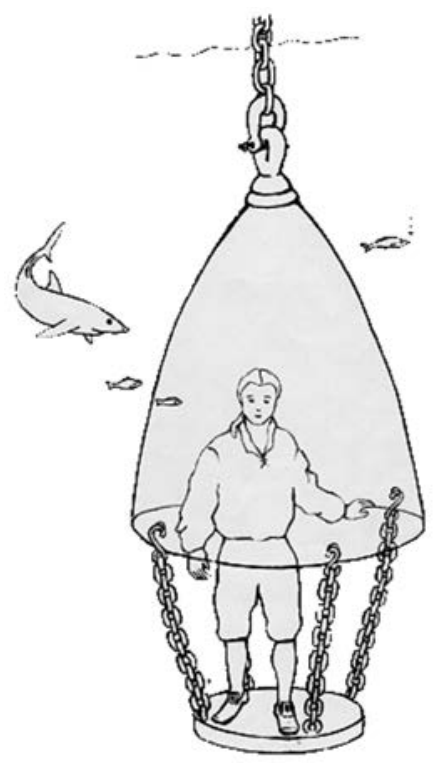

Fig. 1. Una rappresentazione schematica della campana a immersione (da [1]).

In anni recenti, una straordinaria prestazione fu compiuta nel 1913 dal pescatore greco Georgios Haggi Statti, il quale, al largo dell'isola di Scarpanto, nel Dodecanneso da poco divenuto italiano dopo la guerra di Libia, riuscì a liberare e recuperare l'ancora della nave esploratrice italiana Regina Margherita, incagliata alla profondità di 70 metri. A tal fine, egli compì una serie di immersioni in apnea, usando un contrappeso di $15 \mathrm{~kg}$. Al termine, egli sminuì la sua impresa, dichiarando che più volte si era immerso oltre i 100 metri di profondità. Egli disse che a 70 metri, benché la luce sia flebile, ci si vede abbastanza da poter lavorare. Il medico di bordo, che lo visitò, lo trovò bradicardico, enfisematoso, e coi timpani rotti. Il rapporto del medico è riprodotto in [1].

Oggi la pratica professionale dell'apnea si è fortemente ridotta nel mondo intero. Sola eccezione resta quella delle pescatrici coreane e giapponesi, note col nome nipponico di Ama, di cui sono censite ancora alcune migliaia in attività. Ma di queste, molte ormai s'immergono non tanto per la pesca, quanto per accompagnare turisti, pratica ben più redditizia di quella tradizionale. 


\section{LE IMMERSIONI SPORTIVE}

Negli anni recenti invece, è andata sempre più imponendosi la pratica delle immersioni sportive. Originariamente effettuate al solo scopo di stabilire dei primati di profondità, oggi sono anche praticate in competizioni. Dal primo record ufficiale, stabilito da Raimondo Bucher (1912-2008) nel 1949 alla profondità di 30 metri (comunque 40 metri meno di Haggi Statti...), il record non ha mai smesso di evolvere fino a una decina d'anni fa. Ufficialmente, i 50 metri e i 100 metri vennero raggiunti per la prima volta da Enzo Maiorca (1931-2016) nel 1960 e nel 1988, rispettivamente, i 150 metri da Umberto Pelizzari nel 1999.

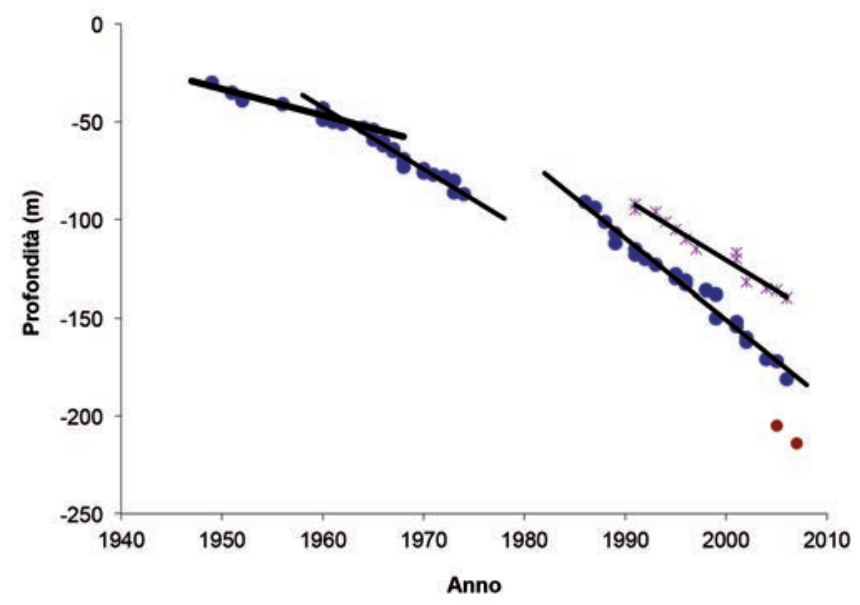

Fig. 2. Evoluzione temporale dei record di profondità durante immersione in apnea. Fino al 1974, si usava la tecnica cosiddetta ad assetto variabile. Dagli anni 1980, si usano le tecniche di immersione libera. Gli asterischi indicano l'evoluzione temporale delle massime profondità raggiunte con la tecnica dell'effetto variabile a partire dagli anni 1980. La distanza verticale rispetto alla funzione che descrive i record con tecnica libera indica l'effetto degli ausili tecnologici. I due punti rossi si riferiscono a due immersioni oltre i 200 metri, di cui quello a destra riguarda l'immersione di Herbert Nietsch con la slitta.

L'evoluzione temporale dei record di profondità è presentata in Fig. 2. Dalla figura appare che, dopo un'epoca diciamo così pionieristica, caratterizzata da una lenta evoluzione dei record, si giunge a una seconda epoca, che si sviluppa nel corso degli anni 1960, in cui le 
immersioni, divenute più rigorose tecnicamente e più professionali, hanno condotto a un più rapido, benché lineare, miglioramento dei record. Segue, a partire dalla metà degli anni 1970, una cesura, causata dal fatto che la Confederazione Mondiale della Attività Subacquee smise di riconoscere i primati d'immersione in apnea per asserite ragioni di sicurezza. Tale blocco venne rimosso a metà degli anni 1980, quando i tentativi ricominciarono. L'evoluzione dei record riprese, sorprendentemente, in maniera lineare, ma la tecnica d'immersione era cambiata, venendo consentite dal nuovo regolamento l'uso di alcuni supporti tecnici, quali le piattaforme di sostegno per la discesa e le mute (o i palloncini) gonfiabili per la risalita. Queste pratiche resero più ergonomiche le immersioni, favorendo il raggiungimento di profondità sempre maggiori (vedi Fig. 3). Queste immersioni vengono oggi chiamate immersioni a tecnica libera.
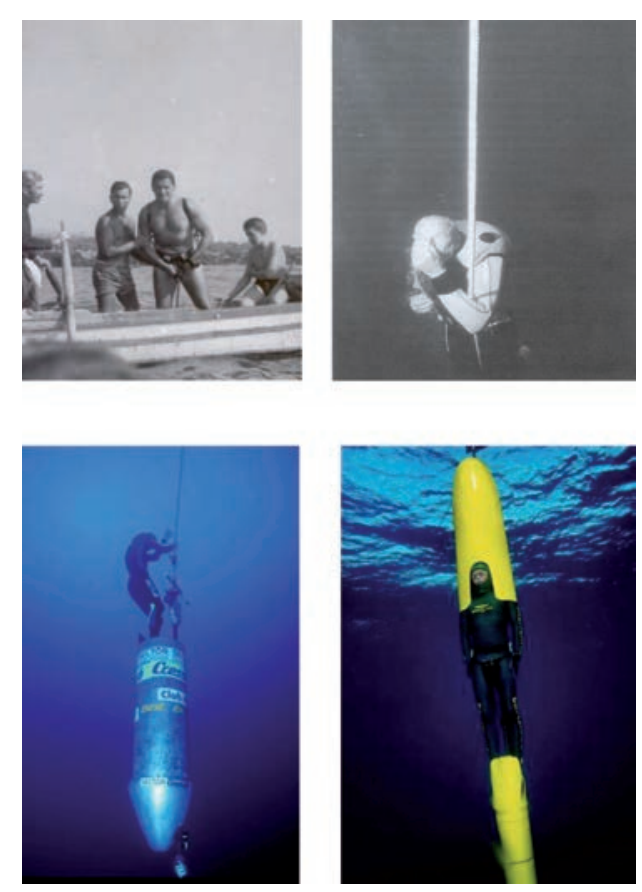

Fig. 3. Da sinistra a destra, e dall'alto in basso: Enzo Maiorca in procinto di immergersi nel 1960; Enzo Maiorca nel 1988, durante l'immersione record a 100 metri; Umberto Pelizzari nel 1999, durante l'immersione record a 150 metri; Herbert Nietsch nel 2007, durante un'immersione con la slitta. 
Il segmento di retta che in Fig. 2 fitta i valori rappresentati da un asterisco si riferisce all'evoluzione del record di profondità nella prova cosiddetta ad assetto variabile. Questa prevede la possibilità di variare l'assetto durante la prova tra discesa e salita (ad esempio, è possibile usare pesi in discesa, lasciandoli a un sommozzatore in attesa sul fondo), ma non di usare i supporti tecnici consentiti nelle immersioni a tecnica libera. La tecnica di immersione ad assetto variabile corrisponde a quella che veniva usata fino a metà degli anni 1970 per i tentativi di record. La distanza verticale tra questo segmento di retta e quello sottostante, statisticamente parallelo, che correla i valori contrassegnati dai simboli blu, indica l'effetto dei nuovi supporti tecnici sulla profondità raggiunta.

Indicare quale sia oggi il record assoluto di profondità mi pare impresa ardua, poiché non vi è consenso al riguardo. La ragione sta nelle immersioni di Herbert Nietsch (Fig. 3), un apneista austriaco che nel 2007 oltrepassò i 200 metri. Tuttavia, egli non si immerse da sé, ma fu calato legato ad una slitta che scorreva lungo un cavo, guidata da un motore elettrico. L'azione del motore incrementò la velocità d'immersione da 1,5 a $4 \mathrm{~m} \mathrm{~s}^{-1}$, riducendo così considerevolmente il tempo di discesa. Questa immersione fu molto contestata, e fu riconosciuta solo dall'associazione privata che la promosse. Da allora, i record assoluti scomparvero dall'attenzione a causa delle controversie regolamentari.

\section{VISIONE CLASSICA DEI LIMITI DELLE IMMERSIONI IN APNEA}

Ambizione dei fisiologi che si occupano di questi argomenti è sempre stata di predire i limiti di profondità per le immersioni in apnea. La prima congettura proposta è un'applicazione della legge di Boyle, che descrive la relazione di proporzionalità inversa tra pressione e volume. Si definisce capacità polmonare totale (CPT) il massimo volume d'aria contenuto nei polmoni, che si raggiunge al termine di una inspirazione massima, e volume residuo (VR) il volume d'aria che rimane nei polmoni al termine di un'espirazione massima. La teoria si basò sul postulato che la $\mathrm{CPT}$ può essere ridotta da un aumento di pressione sul corpo solo fino a diventare uguale al VR. Se le cose stanno così, allora il rapporto tra CPT e VR indica quanto il volume dei polmoni può essere ridotto durante un'immersione per l'aumento di pressione e la legge di Boyle.

Prendiamo come esempio illustrativo il caso delle Ama, le celebri pescatrici coreane e giapponesi. Song et al. [2] misurarono su di esse 
una CPT di 4,58 L e un VR di 1,18 L: il rapporto CPT/VR è uguale a 3,9 , per cui, secondo questa congettura, esse possono raggiungere la profondità alla quale la pressione esterna è quattro volte superiore a quella presente a livello del mare. La loro massima profondità di immersione sarebbe quindi di 30 metri (la pressione aumenta di un'atmosfera per ogni 10 metri di profondità in mare). Poiché esse si immergono per professione fino a un massimo di 20 metri, la loro attività è compatibile con questa congettura.

Fino alla metà degli anni 1960, tutti i record di immersione in apnea erano compatibili con questa congettura. Quando l'americano Bob Croft, nel 1968, stabilì un record a 73 metri, sorsero i primi dubbi, ma la sua $\mathrm{CPT}$ era così alta $(7,8 \mathrm{~L})$, che il suo rapporto $\mathrm{CPT} / \mathrm{VR}$ risultò di 7,7 [3]. Considerato la variabilità intergionaliera di queste misure, si concluse che Croft potesse ancora avere avuto, a 73 metri, un CPT non inferiore al suo VR, ma che un limite estremo era stato raggiunto.

Tuttavia i primati non cessarono di essere migliorati, tanto che non l'esperimento, bensì la pratica condussero alla refutazione della congettura del rapporto CPT/VR. I fisiologi iniziarono a pensare a possibili altri meccanismi, che impedissero il collasso del torace a volumi inferiori al VR. Craig [4] aveva proposto, già nel 1968, che le pressioni negative raggiunte all'interno della gabbia toracica ad elevate profondità, a causa della differente distendibilità di torace e polmone, avrebbero determinato un gradiente di pressione che avrebbe spostato una massa di sangue dalle estremità al torace. In questo modo si sarebbe conseguito un aumento del volume ematico centrale, che avrebbe consentito una riduzione del VR. È la celebre congettura del blood shift. Questa congettura, sovente richiamata all'attenzione negli anni seguenti [vedi ad esempio 5, 6, 7], non ha mai ricevuto evidenza sperimentale diretta. I soli elementi a sostegno di essa sono a tutt'oggi i seguenti: i) la stima che durante immersioni in apnea alla profondità di 40 metri vi possa essere un aumento di volume ematico centrale fi circa $1 \mathrm{~L}$ [3]; ii) l'osservazione che, durante immersione in acqua, circa $700 \mathrm{ml}$ di sangue si spostano verso il torace [8]; iii) la ridotta diminuzione di circonferenza del torace, rispetto a quella dell'addome [9]; iv) segni radiologici di maggiore opacità del torace, indicativi di maggiore presenza in esso di sangue, accompagnati da significativa elevazione del diaframma [10].

Con la continua evoluzione dei record a partire dagli anni 1980, anche questa congettura, comunque relativamente debole benché di ampio successo, fu progressivamente lasciata cadere. 


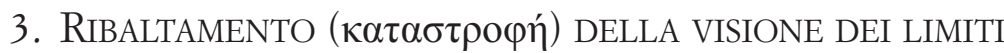 DELLE IMMERSIONI IN APNEA}

Le due congetture qui sopra discusse partivano dal presupposto assiomatico e implicito che i limiti alle profondità massime raggiungibili fossero di natura fisica. Proviamo ora a compiere una rivoluzione assiomatica riguardo alla nostra concezione dei limiti alle immersioni profonde in apnea, affermando che il limite non è di natura fisica, bensì energetica.

Proviamo pertanto a considerare un uomo che si immerge in apnea come un sistema chiuso, che non può scambiare energia, a parte le perdite di calore, con l'ambiente circostante: solo l'energia chimica presente all'interno dell'organismo all'inizio dell'immersione può essere usata per sostenere il metabolismo, che sia a riposo o durante esercizio, e probabilmente nemmeno tutta. Tentiamo ora di costruire una teoria dei limiti delle immersioni profonde in apnea a partire da questi presupposti alternativi.

Quando un essere umano copre una determinata distanza con una determinata modalità di locomozione, la velocità che tale individuo raggiunge è uguale al rapporto tra la potenza metabolica e il costo energetico della locomozione $(\mathrm{C})$. Tuttavia, durante immersioni in apnea, l'obiettivo non è di coprire una data distanza nel più breve tempo possibile, e quindi alla più alta velocità possibile, bensì di percorrere la più lunga distanza possibile. Se questo è lo scopo, allora è conveniente sbarazzarsi del tempo, esprimendo le quantità caratterizzanti il rapporto qui sopra descritto nei termini seguenti: la massima distanza (d) che un essere umano può coprire durante un'immersione in apnea è uguale al rapporto tra l'energia metabolica (E) presente nell'organismo prima dell'immersione e C. Pertanto, d è direttamente proporzionale a E ed inversamente proporzionale a C (Fig. 4). In questa Figura, la pendenza delle linee rette riportate nel grafico superiore è uguale a $\mathrm{C}$, mentre la curvatura delle iperboli equilatere riportate nel pannello inferiore è uguale a $\mathrm{E}$.

Quando ci si muove in acqua, C è uguale a:

$$
\mathrm{C}=\mathrm{D} / \eta
$$

dove $\mathrm{D}$ è la forza che in acqua si oppone all'avanzamento del corpo in movimento (drag in inglese), mentre $\eta$ è l'efficienza meccanica del 
movimento in immersione. Se così è, allora possiamo scrivere che:

$\mathrm{d}=\mathrm{E} / \mathrm{C}=(\mathrm{E} \eta) / \mathrm{D}$
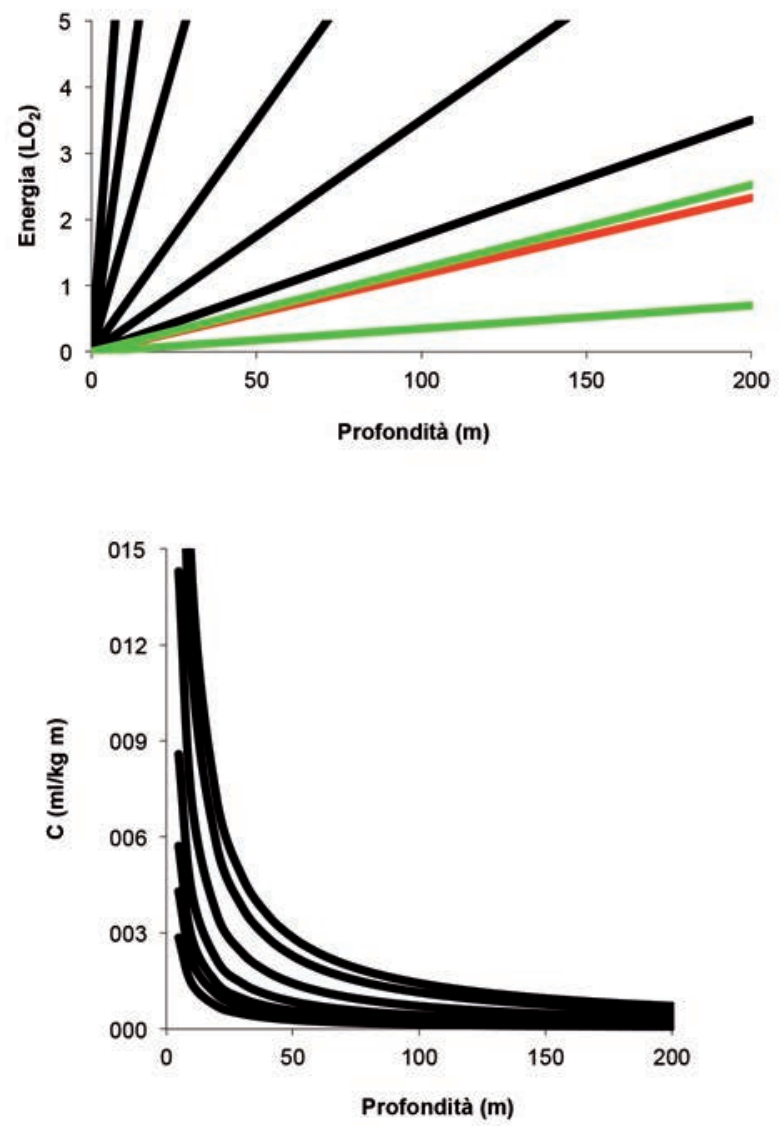

Fig. 4. Rappresentazione grafica teorica della relazione di proporzionalità diretta tra distanza raggiunta (d) e energia metabolica (E) presente nell'organismo prima dell' immersione (pannello superiore), e della relazione di proporzionalità inversa tra de il costo energetico dell'immersione (C) (pannello inferiore). Nel pannello superiore, le linee verdi indicano la relazione per il $C$ equivalente a quello della marcia alla velocità ottimale, e a quello per un'immersione condotta con consumo di ossigeno di riposo. La linea rossa vale per il C stimato da Ferretti et al. [18].

L'equazione 2 ci narra che abbiamo tre possibilità per accrescere $\mathrm{d}$, cioè la massima profondità d'immersione: 1) aumentare la quantità di 
energia di cui disponiamo nell'organismo all'inizio dell'immersione; 2) aumentare il rendimento meccanico dei movimenti in immersione, o del nuoto subacqueo; 3 ) ridurre il drag. La riduzione del drag può essere perseguita migliorando l'idrodinamica dell'immersione. Il miglioramento di $\eta$ si ottiene migliorando le tecniche d'immersione, o del nuoto subacqueo.

Più complesso appare il discorso per quanto riguarda $\mathrm{E}$, poiché negli organismi viventi operano tre forme di metabolismo energetico, cioè i metabolismi aerobico, anaerobico lattacido e anaerobico alattacido, che nel caso delle immersioni in apnea sono sollecitati simultaneamente, e di ciascuno dei quali dobbiamo conoscere la capacità, cioè la massima quantità di energia che ciascuno è in grado di fornire. La massima capacità anaerobica lattacida $\left(\mathrm{E}_{\mathrm{La}}\right.$, dove il suffisso indica il lattato, che è il prodotto terminale di tale via metabolica) e la massima capacità anaerobica alattacida $\left(\mathrm{E}_{\mathrm{PC}}\right.$, dove il suffisso indica la fosfocreatina, che è il principale substrato di tale metabolismo) dettano i limiti dei due metabolismi anaerobici. $\mathrm{E}_{\mathrm{La}}$ è direttamente proporzionale alla massima concentrazione di lattato nel sangue, attraverso una costante detta equivalente energetico del lattato, che vale $3 \mathrm{ml}$ di ossigeno per kg di massa corporea per millimole di lattato accumulata [11]. $\mathrm{E}_{\mathrm{PC}}$ dipende dalla concentrazione intramuscolare di fosfocreatina $(20 \mathrm{mmol}$ per $\mathrm{kg}$ di muscolo umido) [12].

Per quanto riguarda il metabolismo aerobico, durante le immersioni in apnea, poiché non v'è flusso di ossigeno tra ambiente esterno e organismo, la capacità aerobica $\left(\mathrm{EO}_{2}\right)$ dipende dal volume di ossigeno presente nei depositi corporei, che sono i polmoni, il sangue e i tessuti periferici. $\mathrm{Ne}$ deriva che $\mathrm{EO}_{2}$ è uguale alla somma di tre termini, vale a dire:

$\mathrm{EO}_{2}=\mathrm{E}_{\mathrm{L}} \mathrm{O}_{2}+\mathrm{E}_{\mathrm{B}} \mathrm{O}_{2}+\mathrm{E}_{\mathrm{t}} \mathrm{O}_{2}$

Dove i suffissi L, B and t designano i polmoni (lungs), il sangue (blood) e i tessuti (per lo più le fibre muscolari), rispettivamente. $\mathrm{E}_{\mathrm{B}} \mathrm{O}_{2}$ dipende dalla concentrazione ematica di emoglobina, $\mathrm{E}_{\mathrm{t}} \mathrm{O}_{2}$ dalla concentrazione muscolare di mioglobina, e né l'una né l'altra possono essere modificate con manovre ad hoc prima dell'immersione (in effetti, $\mathrm{E}_{\mathrm{B}} \mathrm{O}_{2}$ può aumentare durante un'immersione per contrazione della milza [13], ma poiché non vi sono scambi di ossigeno con l'ambiente esterno, questo non modifica le scorte totali di ossigeno dell'organismo). Pertanto, in prima approssimazione, possiamo semplificare l'equazione (3) come segue 
$\mathrm{EO}_{2}=\mathrm{E}_{\mathrm{L}} \mathrm{O}_{2}+\mathrm{K}$

\section{Dell'importanZa DEI VOLUMi POLMONARI}

L'equazione (4) ci informa di una relazione lineare tra $\mathrm{EO}_{2}$ e $\mathrm{E}_{\mathrm{L}} \mathrm{O}_{2}$, e quindi tra $\mathrm{E} \mathrm{e} \mathrm{E}_{\mathrm{L}} \mathrm{O}_{2}$. La conseguenza pratica è che un apneista può incrementare la durata di un'apnea, e quindi la distanza coperta durante un'immersione profonda (profondità raggiunta) agendo sui volumi polmonari. Pertanto:

1. Gli apneisti estremi hanno volumi polmonari, in particolare la CPT, molto elevati $[14,15,16]$

2. Gli apneisti estremi s'immergono dopo una inspirazione massima, di modo che iniziano le immersioni a partire dalla CPT

3. Tutti fanno qualche manovra di iperventilazione, aumentando per questa via il valore di $\mathrm{E}_{\mathrm{L}} \mathrm{O}_{2}$ a parità di volume.

4. Alcuni praticano anche la respirazione glossofaringea, che è una tecnica respiratoria che consente di introdurre ulteriore aria negli alveoli al di sopra della CPT, con aumento della pressione intrapolmonare e iperdistensione alveolare. Lindholm et al. [15] dimostrarono che con questa tecnica si riescono a introdurre nei polmoni fino a $1,5 \mathrm{~L}$ d'aria in più della CPT.

Ferretti [17] simulò la dinamica della composizione dei gas alveolari durante un'immersione in apnea fino alla profondità di 150 metri, effettuata iniziando con un volume polmonare uguale alla CPT. Egli assunse che l'apneista, prima d'immergersi, effettuasse un'iperventilazione equivalente a quella dei Maiorca e avesse un costo energetico equivalente a quello stimato sui tre Maiorca durante le loro immersioni in mare con tecnica libera [18]. La relazione tra la composizione dei gas alveolari all'emersione e il volume personale, ottenuta con questa simulazione, è riportata in Fig. 5. Questa simulazione indica che Enzo Majorca $(\mathrm{CPT}=7.2 \mathrm{~L})$ e Bob Croft $(\mathrm{TLC}=7.8)$, all'uscita da un'immersione a $150 \mathrm{~m}$ con tecnica libera, avrebbero rispettivamente una $\mathrm{PAO}_{2}$ di 26.1 e $34.6 \mathrm{mmHg}$, e una $\mathrm{PACO}_{2}$ di 41.1 e $39.7 \mathrm{mmHg}$. Una tale composizione dei gas alveolari, quantunque prossima ai limiti del collasso anossico, appare ancora compatibile con uno stato di coscienza all'emersione. Con una CPT del $20 \%$ superiore, simile a quella di Umberto Pelizzari, la composizione dell'area alveolare all'uscita comporterebbe una $\mathrm{PAO}_{2}$ ben superiore $(50 \mathrm{mmHg}$, a cui si associa una 
$\mathrm{PACO}_{2}$ a $37,5 \mathrm{mmHg}$ ), con ampli margini di sicurezza. Con un valore di $\mathrm{C}$ analogo a quello dei Maiorca, una CPT come quella di Pelizzari, rispetto a quella di Maiorca, consentirebbe d'incrementare di 43 metri la massima profondità raggiungibile.

Immersione a $150 \mathrm{~m}$

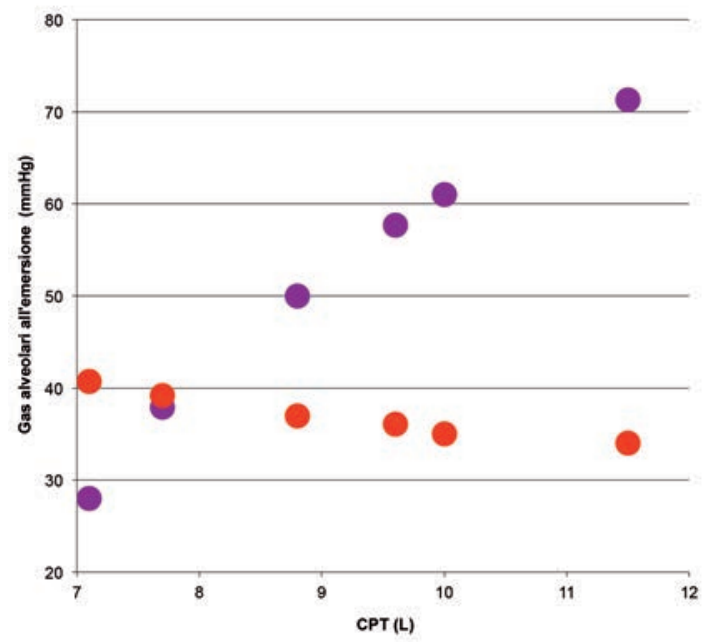

Fig. 5. Relazione teorica, stabilità sulla base di una simulazione di una immersione a 150 $m$, avente costo energetico equivalente a quello stimato su Maiorca durante immersioni sperimentali in mare, tra composizione dei gas alveolari all'uscita (pressione parziale di ossigeno, $P A O_{2}$, in blu, e di anidride carbonica, $P A C O_{2}$, in rosso) in funzione della capacità polmonare totale (CPT). Si assume che le immersioni siano effettuate iniziando con un volume polmonare uguale alla CPT.

\section{L'IMPORTANZA DEL METABOLISMO ANAEROBICO LATTACIDO}

Ferretti et al. [18] dimostrarono anche che, nonostante la bassa potenza metabolica sviluppata durante le immersioni profonde in apnea eseguite con tecnica libera, che si rivela simile a quella della marcia alla velocità ottimale, gli apneisti uscivano dalle immersioni con una concentrazione ematica di lattato superiore a quella di riposo. Ciò aprì la via all'ipotesi che un meccanismo tipo diving response potesse essersi sviluppato anche nell'uomo. Tale ipotesi sarebbe successivamente stata avvalorata da esperimenti condotti in camera iperbarica sui medesimi soggetti [19]. La diving response è un insieme di peculiari risposte cardiovascola- 
ri, caratteristiche di animali che praticano abitualmente immersioni profonde in apnea, che furono identificate originariamente in uccelli marini [20], e successivamente in mammiferi marini quali le foche di Weddel, che vivono in Antartide [21]. La corrente descrizione della diving response può essere riassunta come segue: un'estrema vasocostrizione dei distretti circolatori periferici (muscoli e cute in particolare) genera un aumento possente della pressione arteriosa, a cui segue, forse per meccanismo baroriflesso, un'estrema bradicardia; poiché questo riduce considerevolmente il ritorno venoso al cuore, un aumento di gettata pulsatoria non riesce a compensare il crollo della frequenza cardiaca, cosicché la gettata cardiaca diminuisce di conseguenza. In questa condizione, il sangue viene distribuito solo agli organi vitali, mentre i tessuti periferici vengono esclusi dalla perfusione ematica durante l'immersione. $\mathrm{Ne}$ consegue che essi non ricevono ossigeno per via ematica, per cui devono contare solo sul metabolismo anaerobico, donde l'aumento di concentrazione di lattato all'emersione. Da un punto di vista energetico, la diving response appare quindi come un meccanismo di preservazione dell'ossigeno, che consente di ridurre il consumo di ossigeno, e quindi la caduta di $\mathrm{PAO}_{2}$, e pertanto prolungare la durata dell'immersione. Coerentemente, Lindholm et al. [22] hanno dimostrato che, quanto più intensa è la bradicardia e maggiore l'aumento di pressione, tanto più lenta è la caduta della saturazione del sangue arterioso per l'ossigeno.

Se questa visione è corretta, ne consegue che, per quanto concerne il metabolismo anaerobico lattacido, la $\mathrm{PAO}_{2}$ all'emersione è tanto maggiore, quanto più elevata è la velocità di accumulo del lattato. Ferretti [17] predisse che Enzo Maiorca o Bob Croft non potrebbero emergere coscienti da un'immersione a 150 metri, se la loro velocità di accumulo del lattato for 40 anziché $45 \mu \mathrm{M} \mathrm{s}^{-1}$, né lo potrebbero se, a parità di velocità di accumulo di lattato, la loro potenza metabolica crescesse da 1.00 a $1.05 \mathrm{~L} \mathrm{~min}^{-1}$. L'evenienza di una diving response, stanti le profondità che al giorno d'oggi vengono raggiunte, è ormai una condizione imprescindibile per il successo delle immersioni profonde in apnea.

\section{CONCLUSIONI}

Da questa analisi appare chiaro che, introducendo una teoria energetica dei limiti delle immersioni profonde in apnea, il concetto di profondità limite diviene più modulabile, variando con l'evoluzione 
delle tecniche di immersione e con la variabilità dei molteplici meccanismi fisiologici che determinano i bilanci energetici. In questo contesto, il limite dipende dall'equilibrio tra diversi fattori, in particolare la tecnica di immersione, che agisce sul costo energetico, il rendimento meccanico dei movimenti, che contribuisce, aumentando, a ridurre la potenza metabolica a parità di potenza meccanica, la quantità delle scorte energetiche dell'organismo, donde l'importanza di avere elevati volumi polmonari per raggiungere profondità sempre maggiori, e l'evenienza della diving response, con conseguente incremento del ruolo del metabolismo anaerobico lattacido.

I record di profondità nelle immersioni in apnea continueranno a essere migliorati fintanto che si riuscirà a incrementare le variabili fisiologiche in gioco, ridurre il costo energetico e ottimizzare meglio gli equilibri tra i diversi metabolismi energetici coinvolti.

\section{REFERENZE}

1. Ferretti G, Capelli C. Dagli Abissi allo Spazio: Ambienti e Limiti Umani. Edi Ermes, Milano, 2008.

2. Song SH, Kang DH, Kang BS, Hong SH (1963). Lung volumes and ventilatory responses to high $\mathrm{CO}_{2}$ and low $\mathrm{O}_{2}$ in the Ama. J Appl Physiol 1963: 18: 466-470.

3. Schaefer KE, Allison RD, Dougherty JH jr, Carey CR, Walker R, Yost F, Parker D. Pulmonary and circulatory adjustments determining the limits of depth in breath-hold diving. Science 1968: 162: 1020-1023.

4. Craig AB jr. Depth limits of breath hold diving (an example of Fennology). Respir Physiol 1968: 5: 14-22.

5. Craig AB jr. Depth limits of breath-hold diving. In: The Physiology of BreathHold Diving. Lundgren CEG, Ferrigno M (eds). Undersea and Hyperbaric Medicine Society, Bethesda, MD, USA, pp 9-11, 1987.

6. Lin YC. Physiological limitations of humans as breath-hold divers. In: Man in the Sea. Lin YC, Shida KK (eds). Best, San Pedro, CA, USA, vol. 2, pp 33-56, 1990.

7. Odaglia G. Gli effetti delle alte pressioni sull'organismo. In Sport, Ambiente e Limite Umano. Cerretelli P, di Prampero PE (eds). Mondadori, Milano, pp 185203, 1985.

8. Arborelius M jr, Balldin UI, Lilja B, Lundgren CEG (1972). Hemodynamic changes in man during immersion with the head above water. Aerospace Med 1972: 43: 592-598.

9. Warkander DE, Ferrigno M, Lundgren CEG, McCoy K (1996). Some physiological parameters in a breathhold dive to $107 \mathrm{msw}$ (351 fsw) in the Ocean. Undersea Hyperbaric Med 1996 23: S75. 
10. Morelli L, Data PG. Thoracic radiological changes during deep breath-hold diving (Abstract). In: Proceedings of the Workshop on the Physiology of Deep Breath-Hold Diving. Data PG, Lahiri S (eds). Chieti, Italy, 1991.

11. di Prampero PE, Ferretti G. The energetics of anaerobic muscle metabolism: a reappraisal of older and recent concepts. Respir Physiol 1999: 118: 103-115.

12. di Prampero PE. Energetics of muscular exercise. Rev Physiol Biochem Pharmacol 1981: 89: 143-222.

13. Espersen K, Frandsen H, Lorentzen T, Kanstrup IL, Christensen NJ. The human spleen as an erythrocyte reservoir in diving-related interventions. J Appl Physiol 2002: 92: 2071-2079

14. Ferretti G, Costa M, Moroni R, Ranieri P, Butti F, Sponsiello N. Lung volumes of extreme breath-hold divers. Sport Sci Health 2012: 7: 55-59.

15. Lindholm P, Nyren S. Studies on inspiratory and expiratory glossopharyngeal breathing in breath-hold divers employing magnetic resonance imaging and spirometry. Eur J Appl Physiol 2005: 94: 646-651.

16. Walterspacher S, Scholz T, Tetzlaff K, Sorichter S. Breath-hold diving: respiratory function on the longer term. Med Sci Sports Exerc 2011: 43: 1214-1219

17. Ferretti G. Extreme human breath-hold diving. Eur J Appl Physiol 2001: 84: 254-271.

18. Ferretti G, Costa M, Ferrigno M, Grassi B, Marconi C, Lundgren CEG, Cerretelli P. Alveolar gas composition and exchange during deep breath-hold diving and dry breath holds in elite divers. J Appl Physiol 1991: 70: 794-802.

19. Ferrigno M, Ferretti G, Ellis A, Warkander D, Costa M, Cerretelli P, Lundgren CEG. Cardiovascular changes during deep breath-hold dives in a pressure chamber. J Appl Pbysiol 1997: 83: 1282-1290.

20. Scholander PF. Experimental investigations on the respiratory function in diving mammals and birds. Hvalradets Skrifter, Norske Videnskaps-Akademie Oslo 1940: 22: 1-131.

21. Zapol WM. Diving physiology of the Weddel seal. In: Handbook of Physiology. Environmental Physiology. Fregly MJ, Blatteis CM (eds). Oxford University Press, New York, pp 1049-1056, 1996.

22. Lindholm P, Sundblad P, Linnarsson D. Oxygen-conserving effects of apnea in exercising men. J Appl Physiol 1999: 87: 2122-2127. 
